\title{
Extracorporeal shock wave lithotripsy (ESWL) for large pancreatic stones: are these shocks worth while?
}

\author{
Deepak Kumar Bhasin • Surinder S. Rana
}

Received: 17 July 2010 /Accepted: 28 July 2010 /Published online: 12 August 2010

(C) Indian Society of Gastroenterology 2010

Chronic pancreatitis is characterized by irreversible damage to the pancreas that leads to pain and/or exocrine and endocrine insufficiency [1-3]. Abdominal pain is the most common and distressing symptom and is the most common indication for endoscopic or surgical intervention $[1,4,5]$. One of the mechanisms responsible for pain in chronic pancreatitis is obstruction to the pancreatic duct by strictures or calculi and relief of this obstruction by surgical or endoscopic drainage relieves pain in a majority of patients [6-8]. Endoscopic drainage procedures for relief of pain include pancreatic sphincterotomy, dilation of pancreatic strictures, removal of pancreatic stones and the placement of pancreatic stents to overcome the obstruction [8].

On plain X-ray of the abdomen, pancreatic calcification can be seen in up to $30 \%$ of patients with chronic pancreatitis [4]. The advent of newer imaging modalities like computed tomography (CT) has improved the ability to detect pancreatic calcification [9]. Up to $70 \%$ of patients with alcohol-related chronic pancreatitis can have pancreatic calcification after 10 years of disease [10]. The calcification can be either due to parenchymal or ductal calculi. Pancreatic duct calculi have been reported in $50 \%$ to $90 \%$ of patients with chronic pancreatitis and there is higher incidence of ductal calculi in patients with tropical pancreatitis $[2,11]$.

D. K. Bhasin · S. S. Rana

Department of Gastroenterology,

Post Graduate Institute of Medical Education

and Research (PGIMER),

Sector 12 ,

Chandigarh 160 012, India

D. K. Bhasin $(\bowtie)$

1041, Sector 24-B,

Chandigarh 160 023, India

e-mail: deepakkbhasin@gmail.com

e-mail: dkbhasind@hotmail.com
The ductal calculi cause obstruction leading to an increase in intraductal as well as parenchymal pressure and ischemia, thus causing pain [12]. The factors correlating with pain are the stone size and the diameter of the pancreatic duct.

The goal of endoscopic treatment for chronic painful pancreatitis with ductal calculi is complete clearance of calculi from the duct, thus relieving the obstruction and pain [13-16].

Endoscopic extraction of pancreatic duct calculi is usually more difficult than extraction of bile duct stones because pancreatic stones are generally spiculated, hard and multiple, and on many occasions are impacted behind strictures. Endoscopic sphincterotomy followed by balloon or Dormia-assisted stone extraction is usually successful when the stones are of small size and are located in the head or body of the pancreas, and there is no ductal stricture [17]. The presence of ductal stricture or large calculi creates difficulty in endoscopic clearance and requires either decrease in the size of calculi by breaking them into small pieces or enlarging the ampullary orifice so as to successfully deliver intact large stones.

Endoscopic balloon sphincteroplasty of the papilla has been used to enlarge the papillary orifice and to successfully extract intact ductal calculi $>1 \mathrm{~cm}$, especially radiolucent stones [18]. However, this technique is technically demanding, and has potentially serious complications including bleeding, retroduodenal perforation and pancreatitis [18]. An alternative method is to break the large stones into small pieces so that they can be easily extracted through the papilla. This can be done by using mechanical lithotripsy, intraductal electrohydraulic lithotripsy (EHL) and extracorporeal shock wave lithotripsy (ESWL). Mechanical lithotripsy using a throughthe-scope mechanical lithotripter is technically difficult and is not successful in all cases with large pancreatic calculi, especially when there is difficulty in grasping large stones [19]. Intraductal EHL is cumbersome and requires specialized 
and expensive equipment [20]. ESWL, which works by concentrating focused short waves on stones and causing their disruption, was first used in the field of gastroenterology by Sauerbruch et al. in Germany for fragmentation of gall bladder stones [21]. Thereafter the same authors used it for fragmentation of pancreatic duct stones [22]. Subsequently a number of investigators have used it for fragmentation of pancreatic stones [14-16]. Radiopaque stones can be easily targeted by ESWL under fluoroscopy. Radiolucent stones can be targeted using ultrasound-guided shock wave lithotripsy or by injection of contrast through the nasopancreatic catheter.

After pulverization of the stones by ESWL, the fragmented stones can be retrieved endoscopically. Long-term follow up studies have shown that ESWL combined with endoscopic drainage of the pancreatic duct relieves pain and may avoid the need for surgery in approximately two-thirds of patients [23]. The success rate of pancreatic duct clearance by ESWL varies from $37.5 \%$ to $100 \%$ and pain relief is observed in $79-100 \%$ of cases $[20,24]$ Guda et al. in a meta-analysis described the role of ESWL in the management of chronic calcific pancreatitis [20]. They enrolled 16 studies (none were randomized or case-control studies) with a total of 491 patients, and concluded that ESWL itself, or through facilitation of successful duct clearance endoscopically, leads to improvement of pancreatic pain. Some studies have suggested that breaking of stones to $<2 \mathrm{~mm}$ in size by ESWL alone is effective in providing relief in pancreatic pain as these small stones are spontaneously cleared.

Ohara et al. reported a pilot study showing that the application of ESWL alone led to spontaneous clearance of stone fragments and complete relief of pain in $79 \%$ of patients [25]. Later, a randomized controlled trial also evaluated the efficacy of ESWL alone (26 patients) compared with ESWL plus endoscopic clearance (29 patients) of pancreatic duct stones in patients with painful chronic pancreatitis and dilated pancreatic duct [26]. The authors found that 2 years after treatment, 10 (38\%) patients in the ESWL group had relapse of abdominal pain whereas $13(45 \%)$ in the ESWL plus endoscopy group had relapsed (OR 0.77 ; 95\% CI, $0.23-$ 2.57). One month after treatment, the main pancreatic duct (MPD) diameter as assessed by secretin-MRCP had also significantly decreased in both groups, by a mean of $1.7 \mathrm{~mm}$ (95\% CI 0.9-2.6; $p<0.001$ ); however, the difference between the two groups was not significant. Also by Kaplan-Meier estimates of pain relapse rates for the ESWL alone and combined group were $35.2 \%$ and $42.6 \%$, respectively by 1 year, $39.3 \%$ and $46.7 \%$ by 2 years, and remained stable at $43.3 \%$ and $46.7 \%$ from 3 to 7 years; the pain relapse rates did not differ between the two groups. Importantly, in spite of almost similar effectiveness of therapy in relief of pain, the treatment costs per patient were three times higher in the ESWL combined with endoscopy group compared with the ESWL alone group $(p=0.001)$. The authors concluded that ESWL is a safe and effective preferred treatment for selected patients with painful calcified chronic pancreatitis and combining endoscopy with ESWL adds to the cost of patient care without improving the outcome of pancreatic pain. All these studies have demonstrated that ESWL is a safe technique with low complication rates. The minor complications of ESWL include skin or duodenal contusion, exacerbation of pancreatitis, mild abdominal discomfort and asymptomatic hyperamylasemia. Serious complications reported include hepatic sub-capsular hematoma, splenic rupture, splenic abscess, cholangitis, pancreatitisrelated sepsis, hemosuccus pancreaticus and fluid collections with complication rates ranging from $0 \%$ to $20 \%$ [27].

In India, idiopathic chronic pancreatitis is the most common form of chronic pancreatitis [3, 4] and a study from southern India has shown that pancreatic duct stones in tropical pancreatitis are often large and dense as compared to other etiologies of chronic pancreatitis [28]. It would be interesting to know the effect of ESWL in these large and dense stones. In the current issue of the Journal, Tandan et al. from Hyderabad report a prospective observational study evaluating the efficacy of ESWL in 1,006 patients with chronic calcific pancreatitis (CCP). Out of 1,006 patients, 927 (92.1\%) patients had idiopathic CCP [29]. ESWL was done in patients with calculi $>5 \mathrm{~mm}$ in size; patients with isolated pancreatic tail calculi, extensive calculi in the head, body and tail, multiple MPD strictures, associated pancreatic head mass, pseudocyst, pancreatic ascites and pregnancy were excluded from the study. Pancreatic sphincterotomy was not done prior to ESWL and in patients with radiolucent stones, a naso-pancreatic catheter was inserted prior to ESWL. The authors used a 3rd generation ESWL machine (Delta Compact, Dornier Med Tech., Wessling, Germany) that has both bi-dimensional fluoroscopic and ultrasound targeting facility. Following successful ESWL, the fragments were cleared by ERCP with pancreatic sphincterotomy, which was performed within $48 \mathrm{~h}$ of fragmentation. The authors felt that pancreatic sphincterotomy followed by basketing would clear the pancreatic duct effectively as the calculi in idiopathic pancreatitis are dense. Pancreatic duct stents were placed in all patients with ductal strictures and in patients where duct clearance was considered to be incomplete. The stents were removed within 3 to 6 months after adequate clearance was achieved, and in patients with associated ductal strictures, they were replaced.

Seven hundred and ninety-four patients had multiple ductal calculi and 781 patients had calculi located only in the head region of the pancreas. Following ESWL, the authors could achieve complete clearance of the duct in 762 patients (76.2\%) and partial clearance in 173 patients (17.3\%), which is comparable to earlier published results. Two hundred and ninety-two patients needed one session, 370 patients needed two sessions, 300 patients needed three 
sessions and the rest needed four or more sessions of ESWL for successful fragmentation of the ductal calculi. One hundred and eighty-eight (18\%) patients had associated ductal stricture and stents were placed in 542 (54.2\%) patients. Pancreatic sphincterotomy was done in 938 (93.8\%) patients. ESWL could not fragment the calculi to $<3 \mathrm{~mm}$ diameter or clear the pancreatic duct of $<50 \%$ of stone volume in $71(7 \%)$ patients. Of the 935 successful patients, 846 patients reported for follow up at 6 months. There was significant decrease in the visual analogue score for pain as well as the use of analgesics.

The authors need to be complimented for conducting this study with a large number of patients with CCP and demonstrating that ESWL is effective in pulverizing pancreatic stones in these patients in India also. This study reconfirms the results of the earlier study done by the same group evaluating the effectiveness of ESWL in 250 patients with tropical pancreatitis and multiple radiopaque stones [27]. It has been demonstrated that multiple stones located throughout the duct, stones impacted behind strictures, stones predominantly in the pancreatic tail, or stones associated with complex parenchymal disease such as inflammatory masses do not usually respond well to endoscopic therapy [17], and these patients were excluded from the current study. Thus, the authors rightly chose patients with CCP who were most likely to benefit from endoscopic therapy. In this selected group of 1,006 patients with CCP, ESWL failed in 71 patients. It would be interesting to know the variables that were different in patients who had successful outcome versus those in whom ESWL failed to fragment the stones. Also, as the authors have admitted, 6-month follow up is too short a period to assess the efficacy of any interventional therapy for pain in $\mathrm{CCP}$, as this disease is characterized by remissions and relapses and longer follow up is required to assess the effect of the therapy not only on pain but also on endocrine and exocrine functions of the pancreas. We feel that more studies are required to demonstrate whether in our patients with chronic pancreatitis, ESWL alone is as efficacious as ESWL plus endoscopic drainage, as treatment costs are higher in the ESWL plus endoscopic drainage compared with ESWL alone.

In conclusion, this study emphasizes the fact that any therapy will give best results when the best, most suitable patients are selected. Sherman et al. reported that factors that favored endoscopic removal of stones were stones in the head and body of the pancreas, smaller number of stones, stone diameter less than $10 \mathrm{~mm}$, absence of impacted stone and absence of downstream strictures. Patients with multiple stones required multiple sessions of ESWL and longer duration of treatment $[13,30]$. The most suitable patients for endotherapy of stones in chronic pancreatitis are those who have single or multiple stones located in the main duct without ductal strictures. In such patients, ESWL can help in fragmenting the large stones to small pieces so that can be easily removed endoscopically. Patients with stones located upstream to tight strictures, patients with stones and multiple strictures, patients with stones in the pancreatic tail region and patients with associated inflammatory head mass are usually not suitable candidates for pancreatic endotherapy.

The success of endoscopic therapy for pancreatic stones is dependent on the patient, the performer and the institution. It is worth remembering that each of these has limitations. Therefore, if you chose your patients carefully all these shocks are worth your while.

\section{References}

1. Etemad B, Whitcomb DC. Chronic pancreatitis: diagnosis, classification, and new genetic developments. Gastroenterology. 2001;120:682-707.

2. Ammann RW, Muench R, Otto R, Buehler H, Freiburghaus AU, Siegenthaler W. Evolution and regression of pancreatic calcification in chronic pancreatitis. A prospective long-term study of 107 patients. Gastroenterology. 1988;95:1018-28.

3. Bhasin DK, Singh G, Rana SS, et al. Clinical profile of idiopathic chronic pancreatitis in North India. Clin Gastroenterol Hepatol. 2009;7:594-9.

4. Midha S, Khajuria R, Shastri S, Kabra M, Garg PK. Idiopathic chronic pancreatitis in India: phenotypic characterization and strong genetic susceptibility due to SPINK1 and CFTR gene mutations. Gut. 2010;59:800-7.

5. Witt H, Apte MV, Keim V, Wilson JS. Chronic pancreatitis: challenges and advances in pathogenesis, genetics, diagnosis, and therapy. Gastroenterology. 2007;132:1557-73.

6. Ebbehoj N, Borly L, Bulow J, Rasmussen SG, Madsen P. Evaluation of pancreatic tissue fluid pressure and pain in chronic pancreatitis. A longitudinal study. Scand J Gastroenterol. 1990;25:462-6.

7. Kozarek RA, Patterson DJ, Ball TJ, Traverso LW. Endoscopic placement of pancreatic stents and drains in the management of pancreatitis. Ann Surg. 1989;209:261-6.

8. Warshaw AL, Banks PA. Fernandes-Del Castillo C. AGA technical review: treatment of pain in chronic pancreatitis. Gastroenterology. 1998;115:765-76.

9. Luetmer PH, Stephens DH, Ward EM. Chronic pancreatitis: reassessment with current CT. Radiology. 1989;171:353-7.

10. Ammann RW, Muellhaupt B. The natural history of pain in alcoholic chronic pancreatitis. Gastroenterology. 1999;116:1132-40.

11. Chari ST, Mohan V, Jayanthi V, et al. Comparative study of the clinical profiles of alcoholic chronic pancreatitis and tropical chronic pancreatitis in Tamil Nadu, South India. Pancreas. 1992;7:52-8.

12. Di Sebastiano P, Friess H, Di Mola FF, Innocenti P, Buechler MW. Mechanisms of pain in chronic pancreatitis. Ann Ital Chir. 2000;71:11-6.

13. Sherman S, Lehman GA, Hawes RH, et al. Pancreatic ductal stones: frequency of successful endoscopic removal and improvement in symptoms. Gastrointest Endosc. 1991;37:511-7.

14. Costamagna G, Gabrielli A, Mutignani M, et al. Extracorporeal shockwave lithotripsy of pancreatic stones in chronic pancreatitis: immediate and medium-term results. Gastrointest Endosc. 1997;46:231-6.

15. Adamek HE, Jakobs R, Buttmann A, Adamek MU, Schneider AR, Riemann JF. Long-term follow-up of patients with chronic 
pancreatitis and pancreatic stones treated with extracorporeal shock wave lithotripsy. Gut. 1999;45:402-5.

16. Dumonceau JM, Deviere J, Le Mione O, et al. Endoscopic pancreatic drainage in chronic pancreatitis associated with ductal stones: long-term results. Gastrointest Endosc. 1996;43:547-55.

17. Maydeo A, Soehendra D, Reddy N, Bhandari S. Endotherapy for chronic pancreatitis with intracanalar stones. Endoscopy. 2007;39:653-8.

18. Maydeo A, Bhandari S, Bapat M. Endoscopic balloon sphincteroplasty for extraction of large radiolucent pancreatic duct stones (with videos). Gastrointest Endosc. 2009;70:798-802.

19. Freeman ML. Mechanical lithotripsy of pancreatic duct stones. Gastrointest Endosc. 1996;44:333-6.

20. Guda NM, Partington S, Freeman ML. Extracorporeal shock wave lithotripsy in the management of chronic calcific pancreatitis: A meta-analysis. JOP. J Pancreas (Online). 2005;6:6-12.

21. Sauerbruch T, Delius M, Paumgartner G, et al. Fragmentation of gallstones by extracorporeal shock waves. N Engl J Med. 1986;314:818-22.

22. Sauerbruch T, Holl J, Sackmann M, Werner R, Wotzka R, Paumgartner G. Disintegration of a pancreatic duct stone with extracorporeal shock waves in a patient with chronic pancreatitis. Endoscopy. 1987;19:207-8.

23. Delhaye M, Arvanitakis M, Verset G, et al. Long-term clinical outcome after endoscopic pancreatic ductal drainage for patients with painful chronic pancreatitis. Clin Gastroenterol Hepatol. 2004;2:1096-106.
24. Tadenuma H, Ishihara T, Yamaguchi T, et al. Long term results of extracorporeal shockwave lithotripsy and endoscopic therapy of pancreatic stones. Clin Gastroenterol Hepatol. 2005;3:112835.

25. Ohara H, Hoshino M, Hayakawa $\mathrm{T}$, et al. Single application extracorporeal shock wave lithotripsy is the first choice for patients with pancreatic duct stones. Am J Gastroenterol. 1996;91:1388-94.

26. Dumonceau JM, Costamagna G, Tringali A, et al. Treatment for painful calcified chronic pancreatitis: extracorporeal shock wave lithotripsy versus endoscopic therapy. A randomized controlled trial. Gut. 2007;56:545-52.

27. Ong WC, Tandon M, Reddy V, Rao GV, Reddy DN. Multiple main pancreatic duct stones in tropical pancreatitis: Safe clearance with extracorporeal shockwave lithotripsy. J Gastroenterol Hepatol. 2006;21:1514-8.

28. Chari S, Jayanthi V, Mohan V, Malathi S, Madanagopalan N, Viswanathan M. Radiological appearance of pancreatic calculi in tropical versus alcoholic chronic pancreatitis. J Gastroenterol Hepatol. 1992;7:42-4.

29. Tandan M, Reddy DN, Santosh D, et al. Extracorporeal shock wave lithotripsy and endotherapy for pancreatic calculi-a large single center experience. Indian J Gastroenterol. 2010;29. doi:10.1007/s12664-010-0035-y.

30. Bhasin DK, Poddar U. Long term follow up of patients with chronic pancreatitis and pancreatic stones treated with extracorporeal shock wave lithotripsy. Gastrointest Endosc. 2000;52:586-7. 